\title{
The certainty of uncertainty
}

A recent meeting on climate policy in Madrid ended in failure, as the biggest $\mathrm{CO}_{2}$ polluters, led by the United States, blocked a nonbinding measure intended to help reduce greenhouse gas emissions. Developed nations appear united in their determination not to act on climate change, despite the growing concern of a majority of people around the world. Projections of future fossil fuel use made by nations themselves imply that emissions will keep rising at least through 2030 (https://go.nature.com/364Xgrb). Even supposing that emissions will begin falling then is probably optimistic.

Part of the problem is that self-interested parties know that the costs of inaction will largely fall on people in the future, and those individuals - with the exception of the youth movement inspired by Greta Thunberg lack a voice in the debate. Of course, much of the delay is also the direct result of a decadeslong propaganda campaign funded by fossil fuel interests and right-wing media, which has convinced many people that the science of climate is both unsettled and highly uncertain. Too many people believe that we don't really know much at all, and so we lack any sound basis for action.

Perhaps scientists have been too cautious in countering this view? Over a decade ago, NASA climate scientist James Hansen criticized his fellow scientists for what he called their "scientific reticence". Science requires doubt and self-criticism, as well as brutal honesty about what one knows and does not know. That's a good thing, of course. Yet Hansen argued that the inherently cautious culture of science has led many researchers to express their conclusions about climate change risks in unrealistically mild terms, with too great an emphasis on uncertainties, thereby tempting complacency among the wider public.

Scientists, perhaps, could have spoken more clearly about 'uncertainty', which means one thing in science, and something else in ordinary language. Even in a field as ridden with uncertainty as climate, there are many things we know with high confidence. For example, we have good knowledge of how much global average temperatures will rise for any given increase in $\mathrm{CO}_{2}$. We know less about how much sea level will rise along the United States West Coast, or if rainfall in, say, South China will become more or less predictable.

When it comes to effective communication about uncertainty, research is beginning to help inform on the best strategies. In a recent study, for example, a team of researchers undertook an experiment with more than 1,000 individuals, testing how they responded to messages about climate risks which conveyed uncertainty in different ways. Specifically, they focused on sea level rise. Some individuals were told a single 'most likely' prediction of sea level rise by year 2100 . A second set heard a most likely prediction, augmented with an upper and lower bound. And a third group were told a most likely value, but then heard further information about a 'worst case' scenario, and also a statement of why it is not possible to foresee the full costs and consequences of sea level rise. After all, these depend on other unknown factors such as how much more powerful and frequent future storms turn out to be.

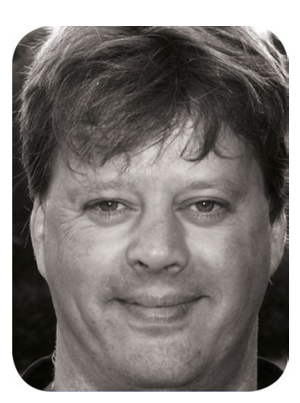

The cautious culture of science leads researchers to express conclusions about climate change in mild terms, tempting complacency among the public.

Perhaps the most positive finding of this study is that people didn't naively prefer the message expressed with no uncertainty. Indeed, the second group hearing the message with upper and lower bounds were the most receptive of the three groups. The researchers suggest that admission of uncertainty made people more likely to trust scientists, as they broadly accept that climate involves inherent uncertainty. Not admitting as much was seen as unrealistic and misleading.

Less encouraging is how people responded to the third message, which, scientifically, might be considered to be conveying the most information. It is certainly true that the full costs of sea level rise are currently almost impossible to assess. They will depend not only on the fine details of the geophysical response to changing climate, but also on the highly uncertain human reaction. Yet this expression of open ended uncertainty tended to undermine listeners' trust, and made them more sceptical. Admission of too much uncertainty seems to make people doubt the science, and they start wondering if the scientists even agree among themselves. People can't comprehend, it seems, how so much uncertainty can be a basis for action.

Scientists tend to react differently, perhaps influenced by other arguments which remain mostly unspoken. For example, they may reason that modern human society has developed largely during the stable climate of the Holocene epoch in the past 10,000 years or so, since the last ice age. Perhaps we can't know for sure how humans will fare in a world driven outside of this climatic band, but we would be entering a zone for which we're historically unprepared. Chances aren't high we'd find those conditions amenable. Or scientists may think that in a system as complex as the climate, with high dimension and innumerable nonlinear feedbacks, the chance of encountering abrupt transitions into violently different climatic conditions is likely high.

So perhaps this study backs up Hansen's point about the danger of scientific reticence. Scientists in public should speak more forcefully about the many climate risks they do know about with considerable certainty. And this ought to include the often unstated reasoning about why it is likely very risky to push our climate away from current conditions. This isn't just conjecture, but a conclusion based on considerable evidence of past climate and evolutionary history, and our knowledge of nonlinear systems.

When it comes to global warming, people know that certainty isn't possible, and scientists gain credibility by openly admitting the limitations of their predictions. They're likely to reach more non-experts by doing so. But there's a danger in going too far. Despite scientists' instincts, they should not hide behind the irreducible complexity of the climate system to avoid a small chance of being wrong. Scientists with an appreciation of climate complexity and the risks of phenomena such as 'tipping points' or critical transitions ought to be more explicit about why our lack of knowledge can sometimes be a reason for action in itself, to avoid catastrophes that seem theoretically too plausible. Without such explicit discussion, this recent study makes clear, arguments for action based on uncertainty simply don't resonate with people.

\section{Mark Buchanan}

Published online: 6 February 2020 https://doi.org/10.1038/s41567-020-0786-Z 